
\title{
25 Research Soure \\ Solitary Fibrous Tumor: A Rare Differential for Submandibular Gland Swelling
}

naveen kumar kushwaha ( $\nabla$ nk8844@gmail.com )

Army Hospital Research and Referral https://orcid.org/0000-0001-9733-0257

\section{Research Article}

Keywords: Submandibular gland, Salivary gland tumor, Soft tissue tumor, Solitary fibrous tumor, CD34 antigen.

Posted Date: January 17th, 2022

DOI: https://doi.org/10.21203/rs.3.rs-1164202/v1

License: (9) This work is licensed under a Creative Commons Attribution 4.0 International License. Read Full License 


\section{Abstract}

\section{INTRODUCTION-}

Solitary fibrous tumour is a spindle cell neoplasm that commonly arise in the pleura and other serosal regions with more than $50 \%$ of these tumours located in the thoracic cavity, however extrathoracic tumours have been reported in various sites such as the liver, skin and the head and neck. Solitary fibrous tumours (SFTs) of the head and neck region are extremely rare with oral cavity being the most common site

\section{MATERIALS AND METHODS-}

A 60 yr. old lady presented with a painless right submandibular swelling that had increased in size during the previous 8 months with no constitutional symptoms. Clinically, there was a firm, mobile, $6 \times 5 \mathrm{~cm}$ right submandibular swelling with no palpable cervical lymph nodes, calculi and the submandibular duct opening was patent. MRI showed a well-defined heterogeneously enhancing lesion involving the right submandibular gland likely neoplastic. FNAC and USG guided core needle biopsy failed to yield any conclusive results. The patient then underwent complete removal of the tumour.

\section{RESULTS-}

Since SFT are rare and share many histological features with other spindle cell neoplasms of the salivary gland, immunohistochemistry is an essential tool to correctly diagnose SFT. NAB2-STAT6 gene fusion product is a highly sensitive and specific marker for SFT and is considered the molecular hallmark. In our case, the resection specimen showed the characteristic histopathological findings for SFT and stained positive for STAT6, CD34, CD99, and BCL-2.

\section{CONCLUSION-}

Clinicians should include SFT in the differential diagnosis of tumours that arise from soft tissues of the head and neck. Immunohistochemical staining is essential to diagnose SFT. Management is complete excision of the mass with long term follow up, because of the potential for recurrence and malignant transformation.

\section{Introduction}

Solitary fibrous tumour is spindle cell neoplasms that commonly arise in the pleura and other serosal regions with more than $50 \%$ of these tumours located in the thoracic cavity, however extrathoracic tumours have been reported in various sites such as the liver, skin and the head and neck. Solitary fibrous tumours (SFTs) of the head and neck region are extremely rare with oral cavity being the most common 
site (1). SFTs are commonly benign, yet $10 \%$ to $15 \%$ of extrapleural SFTs have shown malignant characteristics in the form of metastatic disease (2). SFTs are slow growing masses that can be very difficult to distinguish from other soft tissue tumours, such as synovial sarcoma, benign fibrous histiocytoma, dermatofibrosarcoma protuberans, neurofibroma, schwannoma, fibroma, and myofibroma. The diagnosis is dependent primarily on the microscopic appearance and characteristic immunohistochemical staining. NAB2-STAT6 gene fusion product is a highly sensitive and specific marker for SFT and is considered the molecular hallmark (3). Therefore, definitive diagnosis is usually made after resection of tumor.

\section{Case Report}

A 60 yr. old lady presented with a painless right submandibular swelling that had increased in size during the previous 8 months. The patient denied any related ear, nose, throat and constitutional symptoms. Clinically, there was a right submandibular swelling measuring $6 \times 5 \mathrm{~cm}$ in size. The swelling was mobile and firm in constituency with normal overlying skin. The mass was bimanually palpable, with no palpable calculi and the submandibular duct opening was patent. There were no palpable cervical lymph nodes.

MRI face and neck showed a well-defined heterogeneously enhancing lobulated mass lesion involving the right submandibular gland (predominantly superficial). The lesion appeared heterogeneously hyperintense with multiple cystic/necrotic areas within and hypointense on T1W1. The surrounding fat planes were maintained with no obvious breech in the capsule. Anteriorly and laterally the lesion was causing contour bulge with no involvement of overlying skin or subcutaneous tissue. Posteriorly the lesion was displacing the sternocleidomastoid with no obvious infiltration. Posteromedially the lesion was abutting the right carotid space. Superomedially the lesion was abutting the body of mandible and the intrinsic muscles of the tongue on the right side. No obvious bony erosion was seen. The lesion measured $5.3 \times 5 \times 6.3 \mathrm{~cm}$ (AP $\times \mathrm{ML} \times \mathrm{CC})$. The left submandibular gland appeared normal with no cervical lymphadenopathy. (Fig 1)

The preoperative diagnosis was uncertain because the fine needle aspiration cytology and USG guided core needle biopsy failed to yield any conclusive results. Biopsy showed features of vasoformative neoplasm of uncertain malignant potential. The patient then underwent complete excision of the tumour with right selective node dissection. Intraoperatively, the tumour was found to be confined within its capsule. Macroscopically, the mass was firm and greyish, with a smooth external surface and solid cystic areas. It measured 50 × 50 × 62 mm. Right level II lymph nodes were enlarged. (Fig 2)

On histopathological examination, the tumor is moderately cellular with spindle cells arranged in a haphazard pattern with prominent dilated stag horn like vasculature and areas of collagenisation. The spindle cells displayed plump vesicular nuclei with mild pleomorphism and inconspicuous to conspicuous nucleoli.

Immunohistochemical studies showed that the spindle cells were strongly positive for vimentin, CD99 and CD34 and negative for desmin, Bcl2, smooth muscle actin, cytokeratin, P63, S100. (Fig 3a) The gross 
and microscopic morphological features, supported by the immunohistochemical studies were consistent with the diagnosis of Solitary fibrous tumor (SFT). On modified risk stratification model by Demicco et al, the lesion was categorised as intermediate risk with total score of 4 (4). Right level II lymph nodes were free of tumor. STAT6 immunohistochemical staining was done to confirm the diagnosis. (Fig 3b)

The patient's postoperative course was uneventful, with no evidence of residual or recurrent disease during 1 year follow-up.

\section{Discussion}

SFTs should be managed in the same manner as other soft tissue sarcomas, by a multidisciplinary team consisting of surgeons, medical oncologists, radiation oncologists, and additional ancillary support. Surgical management is the mainstay treatment for SFTs $(4,5)$. It involves wide local excision and preservation of any critical surrounding organs or other structures. Obtaining adequate negative margins has been shown to decrease the rate local disease recurrence and improve survival (6).

While the behaviour of most tumours is benign, a significant fraction of patients will have tumours that demonstrate malignant behaviour. Demicco et al. developed and proposed a risk stratification model to classify patients into low, intermediate and high risk to assess the likelihood of recurrence $(4,7)$. Routine follow up is essential for SFT after surgical resection (6). Oncologic surveillance provides opportunity for early treatment of recurrent or metastatic disease. Given the low incidence of SFT, there are no universal guidelines for surveillance. Surveillance of each patient should be patient specific, dependent on tumor location, risk stratification and other patient factors. Patients age over 55 years, tumor size greater than $15 \mathrm{~cm}$, necrosis, and more than 4 mitoses per ten high power fields suggest more aggressive behaviour (8).

Histologically, a number of other soft tissue neoplasms have a striking resemblance to SFTs. Therefore, differential immunohistochemical staining helps distinguish SFTs from other soft tissue tumours, including benign fibrous histiocytoma, dermatofibrosarcoma protuberans, myofibroma, fibromas, and neurogenic tumours, and other vascular soft tissue tumours, such as hemangiopericytomas and synovial sarcomas. The STAT6-NAB2 fusion product, hallmark of SFT, have been demonstrated by polymerase chain reaction to be present in $91 \%$ of SFT (9).

\section{Conclusion}

SFTs are a rare slow-growing mesenchymal tumor that has been differentiated from other soft tissue tumours over the past decades through advances in histology and molecular genetics. Clinicians should include SFT in the differential diagnosis of tumours that arise from soft tissues of the head and neck. Surgical excision is the mainstay of treatment however long term follow up is required, because of the potential for recurrence and malignant transformation. 


\section{Declarations}

\section{CONFLICT OF INTERESTS}

The authors declare that they have no conflict of interests regarding the publication of this manuscript.

\section{References}

1. Jiang L, Hu C, Chen L, Chen L. Diagnosis of solitary fibrous tumor in the submandibular gland: Case report and literature review. Journal of clinical ultrasound 2019; 47(8).

2. Ridder GJ, Kayser G, Teszler CB, et al. Solitary fibrous tumors in the head and neck: new insights and implications for diagnosis and treatment. Ann Otol Rhinol Laryngol 2007;116:265-70

3. Doyle LA, Vivero M, Fletcher CDM, et al. Nuclear expression of STAT6 distinguishes solitary fibrous tumor from histologic mimics. Mod Pathol 2014;27:390-5.

4. Demicco EG, Park MS, Araujo DM, et al. Solitary fibrous tumor: a clinicopathological study of 110 cases and proposed risk assessment model. Mod Pathol 2012;25:1298-306.

5. Gold JS, Antonescu CR, Hajdu C, et al. Clinicopathologic correlates of solitary fibrous tumors. Cancer 2002;94:1057-68

6. Kayani B, Sharma A, Sewell MD, et al. A review of the surgical management of extrathoracic solitary fibrous tumors. Am J Clin Oncol 2018;41:687-94.

7. Shi W, Wei Z. Solitary fibrous tumor of the submandibular region. Oncol Lett. 2015;9(2):984-986. doi:10.3892/ol.2014.2785

8. El-Naggar AK, Chan JK, Grandis JR, et al. WHO Classification of head and neck tumors (4th edition). IARC: Lyon; 2017

9. Rekhi B, Shetty O, Tripathi P, et al. Molecular characterization of a series of solitary fibrous tumors, including immunohistochemical expression of STAT6 and NATB2-STAT6 fusion transcripts, using reverse transcriptase (RT)-polymerase chain reaction (PCR) technique: an Indian experience. Pathol Res Pract 2017;213:1404-11.

\section{Figures}




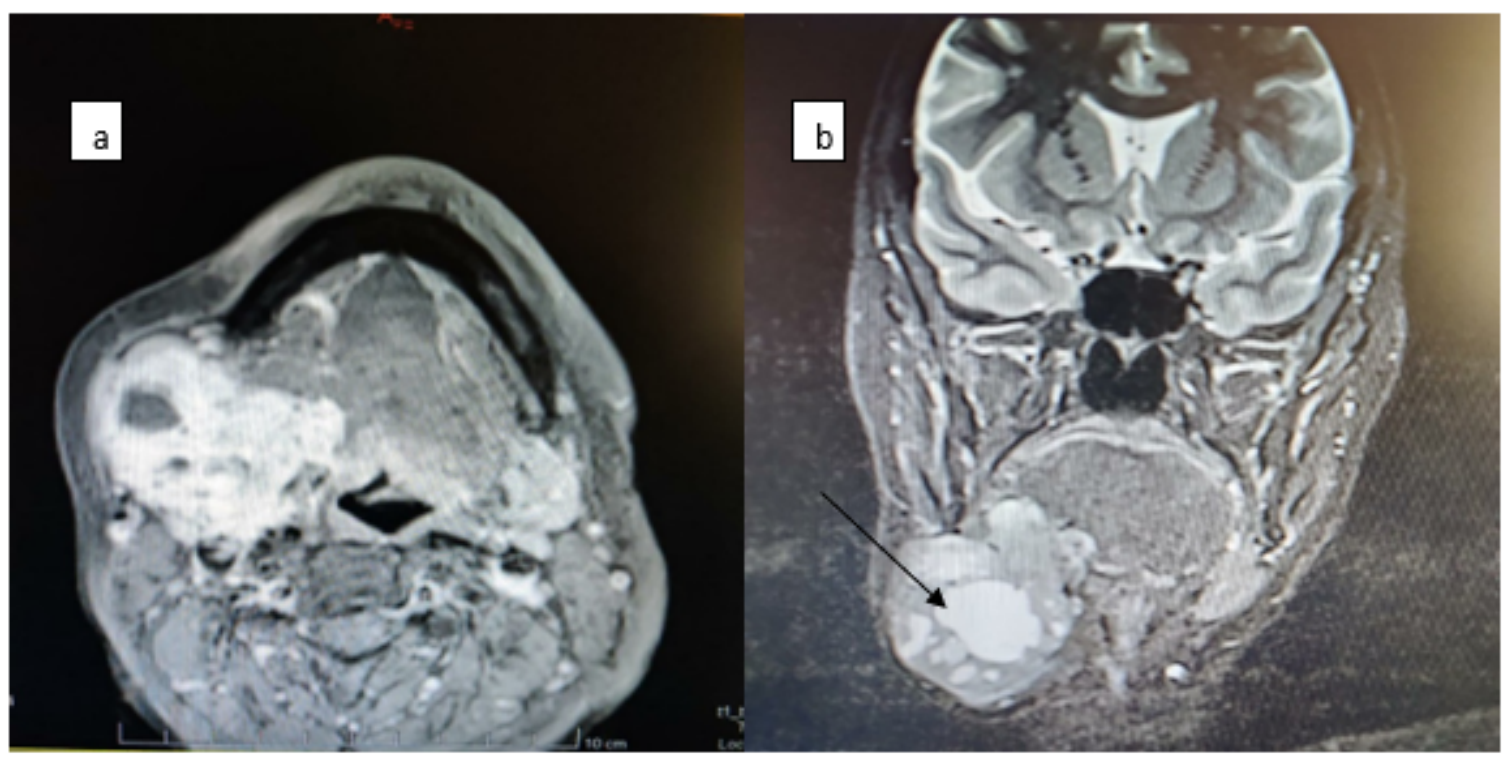

Fig 1: Preoperative MRI face and neck a) Post contrast fast suppressed axial T1W1 b) STIR coronalshowing well defined lobulated mass lesion involving right submandibular gland heterogeneously hyperintense with post contrast enhancement (arrow)

\section{Figure 1}

See image above for figure legend 

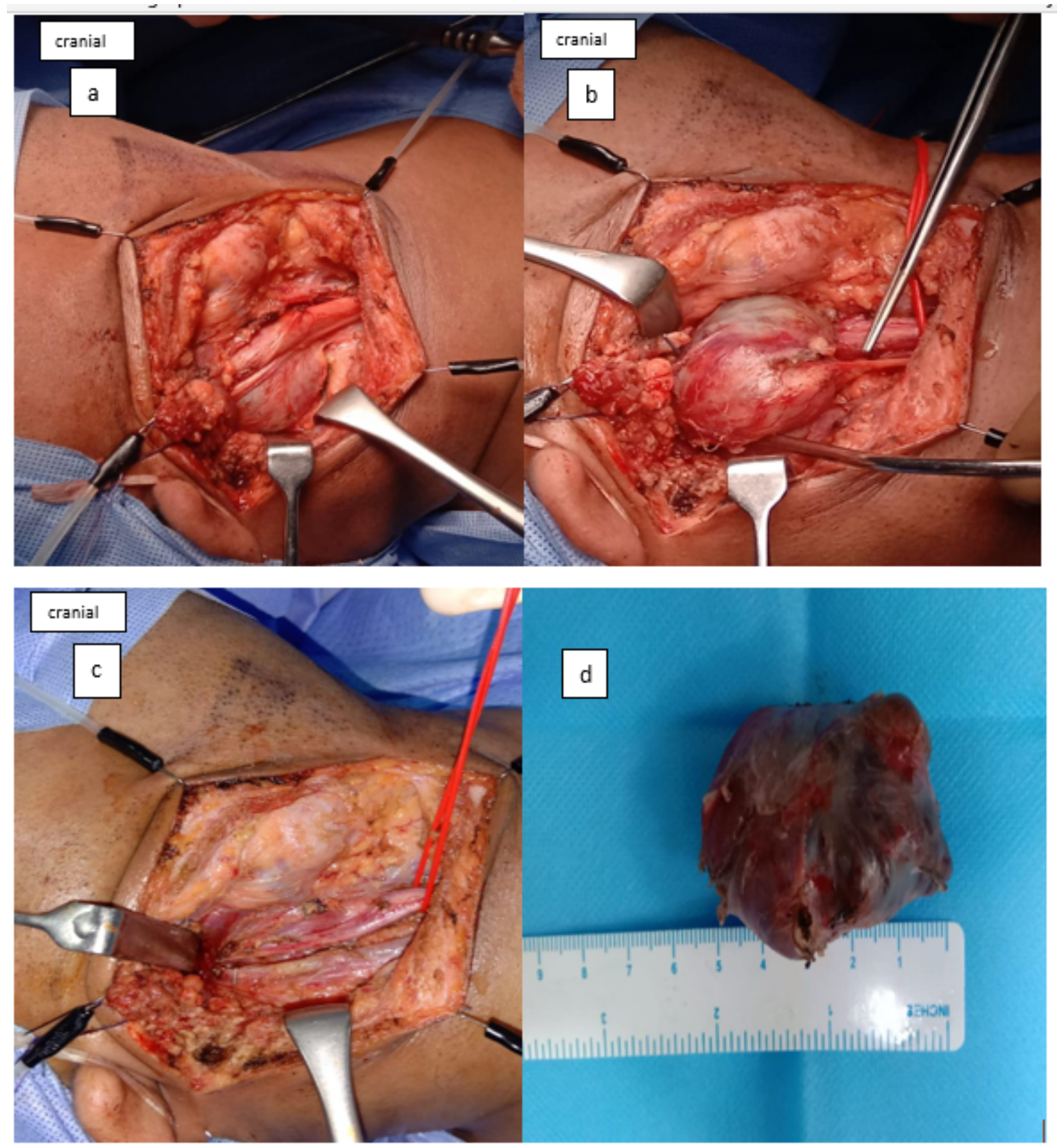

Fig 2: (a-c) Intraoperative images of the right submandibular mass and its relations with surrounding structures (d) gross specimen showing encapsulated tumor of size $5 \times 5 \times 6.2 \mathrm{~cm}$

\section{Figure 2}

See image above for figure legend

\section{Figure 3}

See image above for figure legend 